\title{
Características morfofisiológicas associadas à tolerância à seca em sete genótipos da coleção nuclear de trevo branco
}

\author{
Morphophysiological traits associated with drought tolerance in seven genotypes of the white clover \\ core collection
}

Fernanda Bortolini' ${ }^{I}$ Miguel Dall'Agnol ${ }^{\mathrm{II}}$ Carlos Alberto Bissani $^{\mathrm{II}}$

\section{RESUMO}

\begin{abstract}
O trevo branco é uma leguminosa forrageira muito importante para utilização em pastagens temperadas no Rio Grande do Sul. Entretanto, ele é mais sensivel ao déficit de água no solo do que as outras leguminosas perenes, apresentando problemas de persistência no verão. Com o objetivo de determinar características morfofisiológicas relacionadas à resposta ao déficit hídrico dessa espécie, foi realizado um experimento em casa-de-vegetação, o qual avaliou o efeito da disponibilidade hídrica $(90$ e $40 \%$ da umidade de capacidade de campo do solo) sobre sete acessos pertencentes à Coleção Nuclear de Trevo Branco do Departamento de Agricultura dos Estados Unidos (USDA). O delineamento experimental utilizado foi inteiramente casualizado, com quatro repetições, totalizando 56 vasos. Foram realizadas duas avaliações, aos 130 e aos 196 dias após a semeadura, ocasião em que foram avaliadas diversas características morfofisiológicas. A análise dos resultados indicou que o déficit hídrico teve um efeito marcante sobre a altura de plantas (EST), área foliar (AF), número de folhas vivas (NFV), comprimento de estolão (CES), produção de massa seca (MSPA), taxa fotossintética (A), condutância ( $\mathrm{g}$ ) e eficiência do uso da água (EUA). Nas duas avaliações, em ambas as disponibilidades hídricas, verificaram-se correlações positivas $e$ significativas $(P<0,01)$ entre CES e NFV $(r \geq 0,51)$, assim como entre AF e EST $(r \geq 0,62)$. $O$ acesso 1 foi o mais tolerante ao estresse hídrico, apresentando maior produção de MS sob alta disponibilidade hídrica, e uma das maiores produções de $M S$ sob déficit hídrico. As características NFV e A poderiam ser usadas para a seleção precoce de plantas de trevo branco para tolerância ao estresse hídrico.
\end{abstract}

Palavras-chave: déficit hídrico, estresse hídrico, leguminosas forrageiras, melhoramento vegetal.

\begin{abstract}
The white clover is a forage legume very important for use in temperate pastures in Rio Grande do Sul. However it is more sensitive to water deficit in the soil than other perennial legumes, presenting a lack of persistence in the summer. With the objective of determining morphophysiological traits related to the answer to the water deficit of this species, an experiment was carried out in greenhouse, which evaluated the effect of water availability (90 and $40 \%$ of the soil moisture field capacity) on seven genotypes belonging to the white clover core collection from the United States Department of Agriculture (USDA). The experimental design used was completely random, with four repetitions, totaling 56 pots. Two evaluations were made, at 130 and 196 days after the sowing, when several traits were measured. The analysis of the results indicated that the water deficit had an outstanding effect on most of the variables, such as plant height (PH), foliar area (FA), number of alive leaves (NAL), length of primary stolon (LPS), dry matter of the aerial part (DMAP), photosynthesis rates $(A)$, conductance $(g)$, and water use efficiency (WUE). In both evaluations, regardless the water availability, there were positive and highly significant correlations $(P<0.01)$ between LPS and NAL $(r \geq 0.51)$, as well as between FA and $P H(r \geq 0.62)$. Therefore, the best access under stress was the 1 , which presented larger productions of DMAP at the $90 \%$ level and one of the most productions of DMAP under stress. The NAL and the A are morphological traits that could be used for early white clover selection for tolerance to water stress.
\end{abstract}

Key words: forage legumes, plant breeding, water deficit, water stress.

IEmbrapa Clima Temperado, CP 401, 96010-971, Pelotas, RS, Brasil. E-mail: fernanda.bortolini@cpact.embrapa.br. Autor para correspondência.

IDepartamento de Plantas Forrageiras e Agrometeorologia, Faculdade de Agronomia, Universidade Federal do Rio Grande do Sul (UFRGS), Porto Alegre, RS, Brasil.

IIIDepartamento de Solos, Faculdade de Agronomia, UFRGS, Porto Alegre, RS, Brasil. 


\section{INTRODUÇÃO}

O trevo branco (Trifolium repens L.) é uma das mais importantes leguminosas forrageiras de regiões temperadas do mundo(WILLIAMS, 1987). No RioGrande do Sul, ele apresenta problemas de persistência, principalmente no verão, devido às altas temperaturas e à baixa disponibilidade de água no solo nesse período (PAIM \& RIBOLDI, 1994). Em função da pequena profundidade do sistema radicular de seus estolões e de um baixo controle da transpiração, o trevo branco é mais sensível ao déficit de água no solo do que as outras leguminosas perenes (GARCÍA et al., 2000).

Conforme PIANO \& ANNICCHIARICO (1995), a persistência dos ecótipos de trevo branco está possivelmente associada à densidade de estolões, à produção de massa seca e ao comprimento de entrenó. De acordo com SANDERSON et al. (2003), a persistência dessa espécie nas pastagens deve-se principalmente ao crescimento do estolão. Sob condições de deficit hídrico, a persistência do trevo branco pode ser melhorada pelo aumento da oferta de fósforo às plantas (SINGH \& SALE, 1998). A maior disponibilidade desse elemento promove o aumento do número de folhas pequenas e de ramificações do estolão (SINGH \& SALE, 1997a), além de melhorar a eficiência do uso da água e tolerância à seca de plantas desfolhadas regularmente, pelo decréscimo na taxa de transpiração diária por unidade de área foliar em solo seco (SINGH et al., 2000a).

De acordo com IANNUCCI et al. (2002), as espécies de trevo têm diferente sensibilidade à redução de água no solo, além de apresentarem diferenças em outras características fisiológicas e/ou morfológicas, tais como condutância foliar ou comprimento e profundidade de raízes. Estas são, por consequência, características que necessitam ser estudadas para explicar o desempenho de cada espécie sob estresse hídrico. McMANUS et al. (2000), num estudo comparando as respostas ao déficit hídrico de dois genótipos de trevo branco que compõem o cv. 'Grasslands Kopu' (suscetível à seca) e outros dois que fazem parte da linhagem melhorada 'Syrian selection' (tolerante à seca), verificaram que o pinitol é o açúcar solúvel presente em maior concentração em folhas "maduras" de trevo branco quando sujeitas a um déficit hídrico significativo. Os autores sugeriram ainda que outras características observadas nos genótipos tolerantes como, por exemplo, o pequeno tamanho de folha, deve atuar junto com o aumento da concentração de pinitol, conferindo essa vantagem adaptativa em lugares secos. ANNICCHIARICO \& PIANO (2004) conduziram um estudo a fim de verificar se a densidade de estolão pode ser usada como um critério de seleção indireta para melhorar o desenvolvimento de raiz do trevo branco tipo ladino e os resultados mostraram uma relação positiva entre densidade de estolão e desenvolvimento de raízes. Entretanto, os autores afirmaram não existir nenhuma evidência de uma relação positiva entre desenvolvimento de raiz e tolerância à seca, visto que a massa seca de raízes aumentou quando sob estresse, particularmente em solo de horizonte profundo. Os autores acrescentaram ainda que esse aumento da massa seca de raízes sob estresse foi devido ao crescimento das raízes já existentes, ao invés da produção de novas raízes, pois a seca causou uma redução na porcentagem de nós enraizados.

O objetivo deste trabalho foi determinar características morfofisiológicas relacionadas à resposta ao déficit hídrico em sete acessos pertencentes à Coleção Nuclear de Trevo Branco do Departamento de Agricultura dos Estados Unidos (USDA), juntamente com uma cultivar conhecida ('Ladino Regal'), com características morfológicas contrastantes.

\section{MATERIAL E MÉTODOS}

O experimento foi realizado em ambiente de casa-de-vegetação do Departamento de Plantas Forrageiras e Agrometeorologia da Universidade Federal do Rio Grande do Sul. Foram utilizados sete acessos pertencentes à Coleção Nuclear de Trevo Branco do Departamento de Agricultura dos Estados Unidos (USDA), representando seis países (Tabela 1), os quais apresentaram características contrastantes em relação ao comprimento de estolão, ao tamanho de folíolo (área foliar) e à produção de massa seca em um estudo realizado anteriormente (BORTOLINI et al., 2006).

O substrato utilizado constituiu-se de material de solo proveniente da Estação Experimental Agronômica (EEA), pertencente à unidade de mapeamento São Jerônimo, e classificado como Argissolo Vermelho Distrófico típico - Pvd (STRECK et al., 2002). O material de solo foi seco ao ar, destorroado, peneirado, corrigido, incubado e colocado em vasos de $10 \mathrm{~kg}$. Enquanto o substrato permanecia incubando, foram semeadas 25 sementes de cada acesso diretamente em bandejas olerícolas. As plântulas, após aproximadamente 30 dias da emergência, foram transplantadas para copos plásticos $(500 \mathrm{~mL})$. O experimento foi conduzido de abril a agosto de 2005 , com os tratamentos de disponibilidade hídrica de: 40 e 90\% da umidade de capacidade de campo do solo (CC). Cada unidade experimental foi formada por um vaso, 
Tabela 1 - Identificação dos acessos da Coleção Nuclear de Trevo Branco usados neste estudo.

\begin{tabular}{lcll}
\hline № do acesso & Identificação no USDA* (PI) & País em que foi obtido & Estado de melhoramento \\
\hline 1 & 195534 & Itália & Incerto \\
2 & 214207 & Israel & Desconhecido \\
10 & 419325 & Grécia & Selvagem \\
31 & 282381 & Estados Unidos & Cultivar \\
35 & 269981 & Paquistão & Incerto \\
67 & 239977 & Portugal & Incerto \\
80 & Ladino Regal & Estados Unidos & Cultivar \\
\hline
\end{tabular}

*USDA = Departamento de Agricultura dos Estados Unidos.

dentro do qual foi colocado um saco plástico, $10 \mathrm{~kg}$ do substrato seco, uma planta e quantidade conhecida de água para obter a umidade de capacidade de campo de 40 ou $90 \%$, dependendo do tratamento ao qual pertencia. Cada planta foi inoculada com $1 \mathrm{~mL}$ de uma solução de Rhizobium leguminosarum bv. trifolii (SEMIA 222 e SEMIA 2082, segundo recomendação do Laboratório de Fixação Biológica do Nitrogênio / Fundação Estadual de Pesquisa Agropecuária / LFBN / FEPAGRO / Porto Alegre) em solução aquosa (concentração de $10^{9}$ células $\mathrm{mL}^{-1}$ ). Cada vaso foi pesado e regado diariamente, sendo as avaliações morfofisiológicas realizadas aos 130 e aos 196 dias após a semeadura. Na primeira avaliação (não-destrutiva), foram medidas as seguintes características: altura de plantas (EST), comprimento de estolão primário(CES1), utilizando uma régua graduada $(\mathrm{cm})$; hábito de crescimento (HAB), avaliado visualmente, sendo atribuídas notas 1,2 e 3 para plantas prostradas, decumbentes e eretas, respectivamente; número de folhas vivas (NFV) e senescentes (NFS), por contagem; área foliar de 3 folhas (AF), em um planímetro ótico modelo LICOR 3100; e produção de massa seca da parte aérea (MSPA = folha + estolão), através do corte das plantas com auxílio de tesouras e secagem em estufa a $60^{\circ} \mathrm{C}$ até massa constante. Por outro lado, na segunda avaliação (destrutiva), foram também avaliados: comprimento $(\mathrm{cm})$ de estolão secundário (CES2) e terciário (CES3), número de ramificações por estolão (rami/est), número de inflorescências (NI) por contagem; produção de massa seca de folha (MSF), de estolão (MSE) e de raiz (MSR), através do corte das plantas com auxílio de tesouras, e secagem em estufa a $60^{\circ} \mathrm{C}$ até massa constante; taxa fotossintética líquida (A), condutância estomática (g), transpiração (Tr), através de um medidor de fotossíntese modelo LICOR 6400, e eficiência do uso da água (EUA), através da razão entre fotossíntese e transpiração. O delineamento experimental utilizado foi inteiramente casualizado, com quatro repetições. A análise estatística foi realizada com auxílio do programa SAS 8.2 (SAS Institute, 2001), constituindo-se de uma análise de variância (one way) e da comparação das médias pelo teste de Tukey a 5\% de probabilidade. Posteriormente, foi realizada a análise de correlação simples para cada tratamento dentro de cada avaliação, utilizando-se quatro repetições por acesso para cada uma das 18 características avaliadas. Finalmente, foram gerados índices de tolerância para AF e MSPA para os dois cortes, dividindo-se os valores obtidos a $40 \%$ por aqueles obtidos a $90 \%$ da CC.

\section{RESULTADOS E DISCUSSÃO}

A análise estatística dos resultados obtidos indicou que, na primeira avaliação, todas as características avaliadas foram afetadas pela disponibilidade de água, embora os acessos avaliados não tenham diferido significativamente quanto à produção de forragem dentro de cada disponibilidade hídrica. Na segunda avaliação, houve interação genótipo x níveis de água para altura de plantas (EST), número de folhas vivas (NFV), comprimento de estolão primário (CES1), área foliar (AF), massa seca de folha (MSF), massa seca de estolão (MSE), número de ramificações por estolão (ramif/est), condutância estomática $(\mathrm{g})$, transpiração $(\mathrm{Tr})$ e número de inflorescências (NI). Contudo, não houve diferença significativa entre os acessos na menor disponibilidade hídrica (40\% da CC) para nenhuma das características avaliadas. Observou-se ainda que na segunda avaliação os acessos diferiram significativamente quanto à produção de MS a $90 \%$ da $\mathrm{CC}$, sendo os acessos 1, 31 e 35 os que apresentaram maior MSPA, enquanto que os acessos 1, 35 e 67 foram os que reduziram significativamente o CES1 quando sob estresse. Esses resultados concordam com aqueles obtidos por LUCERO et al. (1999), os quais afirmaram que ambos os rendimentos de massa seca e estolão de trevo branco diminuíram com o aumento do déficit hídrico no solo.

Ciência Rural, v.41, n.9, set, 2011. 
Dentre os acessos com maior produção de forragem, $\mathrm{o}$ acesso 1 caracterizou-se por uma maior AF e MSF, enquanto que o 35, pelos maiores CES, MSE, rami/est e NFV, evidenciando a importância do estolão e da AF na ausência de déficit hídrico. O acesso 10, juntamente com o 80 , foram os que apresentaram menor MSPA, sendo que o primeiro também apresentou menor $\mathrm{AF}$ e EST e maior rami/est. Com exceção do acesso 10, todos os demais reduziram significativamente a AF sob estresse, apresentando, como no caso do acesso 1 na segunda avaliação, valores até cinco vezes menores do que na ausência do déficit hídrico (Tabela 2).

Observando-se a figura 1, nota-se que a taxa fotossintética foi reduzida praticamente à metade no tratamento a $40 \%$ da CC para a maioria dos acessos, havendo diferença significativa entre os tratamentos hídricos. Segundo LAWLOR (1995), as possíveis razões para esse decréscimo na taxa fotossintética quando as plantas são submetidas ao déficit hídrico decorrem da limitada difusão de $\mathrm{CO}_{2}$ para os espaços intercelulares da folha, como consequência da redução estomática, e o metabolismo prejudicado devido à inibição direta de processos bioquímicos pela perda de água da célula. Os acessos 35 e 67 apresentaram maiores taxas fotossintéticas e menores condutâncias e transpiração, quando submetidos à alta disponibilidade hídrica, utilizando a água de forma mais eficiente a $90 \%$ da CC. Entretanto, sob o estresse, o acesso 67 foi o que apresentou menor taxa fotossintética, reduzindo também a condutância e transpiração. Por sua vez, o acesso 31, que apresentou uma das maiores taxas fotossintéticas, juntamente com menores taxas de condutância e transpiração a $40 \%$ da CC, foi o que teve maior eficiência do uso da água durante o estresse hídrico (Figura 1).

Nas duas avaliações, em ambas as disponibilidades hídricas, verificaram-se correlações positivas e altamente significativas $(\mathrm{P}<0,01)$ entre CES e NFV ( $r \geq 0,51)$, assim como entre AF e EST $(r \geq 0,62)$. Observando-se a tabela 3 , verifica-se que, na primeira avaliação, no tratamento a $40 \%$ da CC, a MSPA apresentou correlação positiva e altamente significativa com a $\mathrm{AF}(\mathrm{r}=0,50)$ e o NFV ( $r=0,61)$, enquanto que, no tratamento a $90 \%$ da CC, a MSPA não se correlacionou significativamente com a AF, mas sim ao CES1 $(\mathrm{r}=0,57)$, juntamente com o NFV $(r=0,61)$ e a EST $(r=0,47)$. Na segunda avaliação (dados não apresentados), as características que apresentaram correlação positiva e altamente significativa com a MSPA, no tratamento a $40 \%$ da CC, foram oCES1 $(r=0,79)$ e o NFV $(r=0,87)$ e, a $90 \%$ da CC, apenas o CES1 ( $r=0,71)$. Esse fato pode indicar que, no primeiro corte, quando as plantas estavam menores, a MSPA foi correlacionada com a $\mathrm{AF}$, enquanto que, no segundo corte, como as plantas já estavam maiores, o CES1 passou a ter importância na formação da MSPA. Verificou-se também alta correlação entre MSPA e fotossíntese $(r=0,89)$, indicando que quanto maior a MSPA, maior era a taxa fotossintética dos acessos. O número de ramificações por estolão está correlacionado com os CES1, CES2 e CES3, com o NFV $(r=0,65)$ e negativamente correlacionado com a $\mathrm{AF}(\mathrm{r}=-0,45)$, indicando que quanto maior o CES, maior o NFV e menor a AF dessa planta. O número de inflorescências está correlacionado com a MSF $(r=0,60)$ e a $\operatorname{MSPA}(r=0,48)$, mostrando que quanto maior a MSF e MSPA, mais inflorescência as plantas apresentavam. Houve correlação negativa entre tamanho de folha e taxa de transpiração $(r=-0,10)$, embora ela não tenha sido alta e significativa tal como relatado anteriormente por SINGH et al. (2000a).

A MSPA total (soma dos dois cortes) foi correlacionada com NFV no tratamento a $40 \%$, enquanto que a $90 \%$ foi correlacionada com o CES. Isso indica que, sob déficit hídrico, o NFV foi importante para a formação da MSPA, apresentando menor

Tabela 2 - Médias dos acessos de Trevo Branco para as disponibilidades hídricas 40 e 90\% e índices de tolerância para AF nas duas avaliações, MSPA no segundo corte e MSPA total (soma dos dois cortes).

\begin{tabular}{|c|c|c|c|c|c|c|c|c|c|c|c|c|}
\hline \multirow{2}{*}{ Ac } & \multicolumn{2}{|c|}{$---A F 1\left(\mathrm{~cm}^{2}\right)---$} & \multirow{2}{*}{ Índice } & \multicolumn{2}{|c|}{$---A F 2\left(\mathrm{~cm}^{2}\right)---$} & \multirow{2}{*}{ Índice } & \multicolumn{2}{|c|}{---MSPA c2--- } & \multirow{2}{*}{ Índice } & \multicolumn{2}{|c|}{--MSPA tot-- } & \multirow{2}{*}{ Índice } \\
\hline & $40 \%$ & $90 \%$ & & $40 \%$ & $90 \%$ & & $40 \%$ & $90 \%$ & & $40 \%$ & $90 \%$ & \\
\hline 1 & 7,33 & 18,88 & $0,39 \mathrm{~b}$ & 4,08 & 22,97 & $0,18 \mathrm{a}$ & 0,37 & 5,48 & $0,07 \mathrm{a}$ & 0,46 & 5,83 & $0,08 \mathrm{a}$ \\
\hline 2 & 8,95 & 16,88 & $0,53 \mathrm{~b}$ & 4,42 & 15,41 & $0,29 \mathrm{a}$ & 0,40 & 2,25 & $0,18 \mathrm{a}$ & 0,53 & 2,51 & $0,21 \mathrm{a}$ \\
\hline 10 & 4,69 & 3,80 & $1,23 \mathrm{a}$ & 2,32 & 3,74 & $0,62 \mathrm{a}$ & 0,43 & 1,28 & $0,33 \mathrm{a}$ & 0,55 & 1,46 & $0,37 \mathrm{a}$ \\
\hline 31 & 8,87 & 16,17 & $0,55 \mathrm{~b}$ & 5,11 & 15,43 & $0,33 \mathrm{a}$ & 0,24 & 4,93 & $0,05 \mathrm{a}$ & 0,32 & 5,31 & $0,06 \mathrm{a}$ \\
\hline 35 & 4,21 & 8,15 & $0,52 \mathrm{~b}$ & 2,36 & 6,31 & $0,37 \mathrm{a}$ & 0,15 & 4,63 & $0,03 \mathrm{a}$ & 0,22 & 5,07 & $0,04 \mathrm{a}$ \\
\hline 67 & 7,05 & 14,20 & $0,50 \mathrm{~b}$ & 4,03 & 9,99 & $0,40 \mathrm{a}$ & 0,13 & 3,57 & $0,04 \mathrm{a}$ & 0,24 & 3,99 & $0,06 \mathrm{a}$ \\
\hline 80 & 7,39 & 12,93 & $0,57 \mathrm{~b}$ & 6,21 & 13,62 & $0,46 \mathrm{a}$ & 0,14 & 2,26 & $0,06 \mathrm{a}$ & 0,22 & 2,55 & $0,09 a$ \\
\hline
\end{tabular}

Médias seguidas de mesma letra na coluna não diferem significativamente pelo teste de Tukey a 5\%.

Ciência Rural, v.41, n.9, set, 2011. 


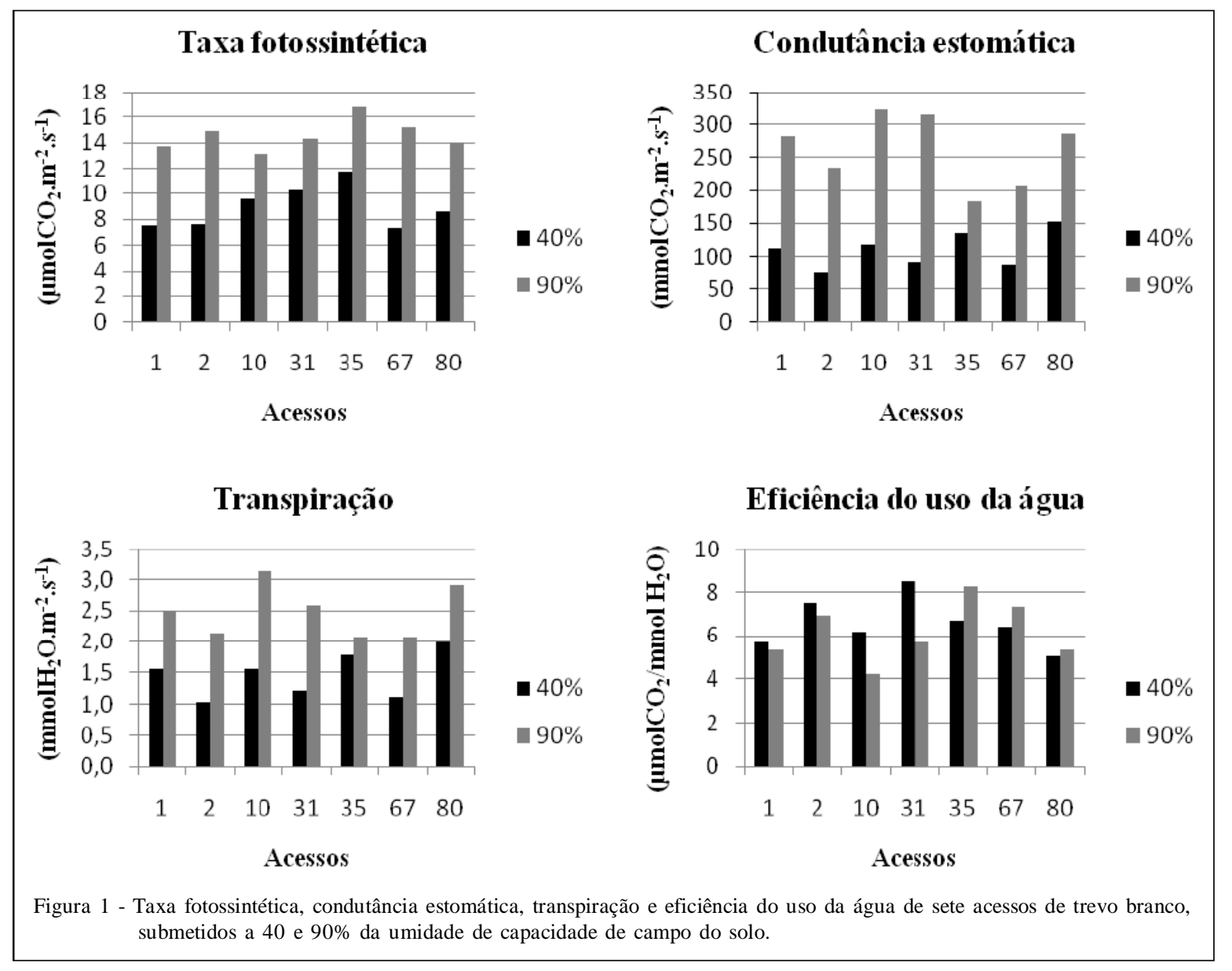

importância sob alta disponibilidade de água, já que o CES foi o maior responsável pela produção de MSPA.

Os índices de tolerância são usados para tornar possível a comparação de tolerância entre diferentes estações e/ou entre diferentes espécies por igualar as grandezas. Observando-se a tabela 2, podese notar que os índices de AF diferiram significativamente na primeira avaliação enquanto que os de MSPA, tanto para o primeiro (dados não mostrados) como para o segundo corte, não apresentaram diferença significativa entre os acessos. De acordo com os resultados de AF, o acesso mais tolerante foi o acesso 10, o qual apresentou o maior índice $(1,23)$. No entanto, verificando os valores de AF desse acesso, percebe-se que esse é o acesso com menor AF tanto no tratamento a 40, quanto a $90 \%$ da $\mathrm{CC}$, apresentando menor produção de forragem nas duas avaliações. Em contrapartida, os acessos 1, 2 e 31 apresentam índices mais baixos, mas maior AF em ambos os tratamentos hídricos, produzindo maior quantidade de forragem, que seria mais interessante nesse caso.
Quanto à produção de MSPA (Tabela 2), os índices não diferiram significativamente em nenhum dos dois cortes. Levando-se em conta essa análise, sem verificar os valores absolutos de produção, a seleção de materiais mais tolerantes pode resultar em plantas com baixa produção de MS, por exemplo, como observado com os índices para MSPA total. O acesso 10 apresentou maior índice $(0,37)$, sendo o acesso mais tolerante, mas apresentou MSPA total menor do que os demais no tratamento a $90 \%$ e igual produção a $40 \%$ da CC, Além disso, o acesso 1, que apresentou maior MSPA total, possui um dos menores índices $(0,08)$, mas sua produção foi muito maior a $90 \%$ da CC, sendo mais desejável nesse caso.

Portanto, de acordo com os resultados obtidos, a utilização de índices de tolerância não é uma boa ferramenta para a seleção, uma vez que se procuram acessos com boa produção de forragem sob a condição de estresse, evitando-se acessos altamente tolerantes, mas que não produzam forragem de maneira satisfatória. 
Tabela 3 - Correlações entre as características na primeira avaliação, nos tratamentos a 40 e $90 \%$ da CC.

\begin{tabular}{|c|c|c|c|c|c|c|c|}
\hline & EST & NFV & NFS & CES & $\mathrm{AF}$ & MSPA & MSPAtotal \\
\hline \multicolumn{8}{|l|}{$40 \%$} \\
\hline EST & & $-0,19$ & $-0,21$ & $-0,04$ & $0,62 * *$ & 0,25 & $0,38^{*}$ \\
\hline NFV & & & $0,39 *$ & $0,51 * *$ & $-0,13$ & $0,61 * *$ & $0,50^{* *}$ \\
\hline NFS & & & & 0,11 & $-0,21$ & 0,20 & $-0,13$ \\
\hline CES & & & & & $-0,16$ & 0,28 & 0,31 \\
\hline $\mathrm{AF}$ & & & & & & $0,50 * *$ & $0,37^{*}$ \\
\hline MSPA & & & & & & & $0,47 * *$ \\
\hline \multicolumn{8}{|l|}{ MSPAtotal } \\
\hline \multicolumn{8}{|l|}{$90 \%$} \\
\hline EST & & $-0,09$ & & $-0,07$ & $0,85^{* *}$ & $0,47 * *$ & $0,50^{* *}$ \\
\hline NFV & & & & $0,65^{* *}$ & $-0,21$ & $0,61 * *$ & 0,28 \\
\hline CES & & & & & $-0,13$ & $0,57 * *$ & $0,42^{*}$ \\
\hline $\mathrm{AF}$ & & & & & & 0,33 & $0,39 *$ \\
\hline MSPA & & & & & & & $0,55^{* *}$ \\
\hline MSPAtotal & & & & & & & \\
\hline
\end{tabular}

* Significativo a $5 \%$ de probabilidade pela estatística $\mathrm{t}(\mathrm{P}<0,05)$.

**Significativo a $1 \%$ de probabilidade pela estatística $\mathrm{t}(\mathrm{P}<0,01)$.

\section{CONCLUSÃO}

O déficit hídrico teve um significante efeito sobre as características altura, área foliar, número de folhas vivas, comprimento de estolão, produção de massa seca da parte aérea, taxa fotossintética, condutância e eficiência do uso da água. $\mathrm{O}$ acesso 1 foi o mais tolerante ao estresse hídrico, apresentando maior produção de massa seca sob alta disponibilidade hídrica, e uma das maiores produções de massa seca sob déficit hídrico. A princípio, as características número de folhas vivas e taxa fotossintética poderiam ser usadas para a seleção precoce de plantas de trevo branco para tolerância ao estresse hídrico.

\section{AGRADECIMENTOS}

Ao Conselho Nacional de Desenvolvimento Científico e Tecnológico (CNPq), pela bolsa de estudos concedida ao primeiro autor, ao Departamento de Agricultura dos Estados Unidos (USDA), pelo fornecimento das sementes, e aos bolsistas de Iniciação Científica e colegas mestrandos, pela ajuda nas avaliações, pesagens e tabulação de dados, em especial ao Armando Martins dos Santos, pelo grande auxílio com a análise estatística.

\section{REFERÊNCIAS}

ANNICCHIARICO, P.; PIANO, E. Indirect selection for root development of white clover and implications for drought tolerance. Journal of Agronomy and Crop Science, v. 190, n.1, p.28-34, 2004. Disponível em: <http://onlinelibrary.wiley.com/ doi/10.1046/j.0931-2250.2003.00070.x/full>. Acesso em: 5 jul. 2011. doi: 10.1046/j.0931-2250.2003.00070.x.
BORTOLINI, F. et al. Caracterizações morfológica e agronômica e divergência genética em germoplasma de trevo branco. Revista Brasileira de Zootecnia, v.35, n.4, suppl., p.1601-1610, 2006. Disponível em: <http://www.scielo.br/ s cie lo.php? s cript $=$ sci_art text \& pid=S 1516 $35982006000600005 \& \operatorname{lng}=\mathrm{en} \& \mathrm{nrm}=\mathrm{iso} \& \ln \mathrm{ln}=\mathrm{pt}>$. Acesso em: 17 jun. 2011. doi: 10.1590/S1516-35982006000600005.

GARCÍA, J.A. et al. Riego y producción de semillas de trébol blanco. In: JORNADA DE TREBOL BLANCO, 2000, Estanzuela. [Anais...] Estanzuela: INIA, 2000. p.13-18. (Serie Actividades de Difusión, 241).

IANNUCCI, A. et al. Water deficit effects on osmotic adjustment and solute accumulation in leaves of annual clovers. European Journal of Agronomy, v.16, n.2, p.111-122, 2002. Disponível em: <http://www.sciencedirect.com/science/article/ pii/S1161030101001216>. Acesso em: 5 jul. 2011. doi: 10.1016/S1161-0301(01)00121-6.

LAWLOR, D.W. The effects of water deficit on photosynthesis. In: SMIRNOFF, N. (Ed). Environment and plant metabolism: flexibility and acclimation. Oxford: BIOS Scientific Publishers, 1995. p.129-160.

LUCERO, D.W. et al. Effects of water deficit and plant interaction on morphological growth parameters and yield of white clover (Trifolium repens L.) and ryegrass (Lolium perenne L.) mixtures. European Journal of Agronomy, v.11, n.3-4, p.167-177, 1999. Disponível em: <http:// ww w.sciencedirect.com/science/article/pii/ S1161030199000283>. Acesso em: 5 jul. 2011. doi: 10.1016/ S1161-0301(99)00028-3.

McMANUS, M.T. et al. Pinitol accumulation in mature leaves of white clover in response to a water deficit. Environmental and Experimental Botany, v.43, n.1, p.11-18, 2000. Disponível em: <http://www.sciencedirect.com/science/article/ pii/S0098847299000416>. Acesso em: 5 jul. 2011.doi: 10.1016/ S0098-8472(99)00041-6.

Ciência Rural, v.41, n.9, set, 2011. 
PAIM, N.R.; RIBOLDI, J. Duas novas cultivares de trevo branco comparadas com outras disponíveis no Rio Grande do Sul, em associação com gramíneas. Pesquisa Agropecuária Brasileira, v.29, n.1, p.43-53, 1994. Disponível em: <http:/ /webnotes.sct.embrapa.br/pab/pab.nsf/FrAnual>. Acesso em: 5 jul. 2011.

PIANO, E.; ANNICCHIARICO, P. Persistence of ladino white clover ecotypes and relationship with other agronomic traits. Grass Forage Science, v.50, n.3. p.195-198, 1995. Disponível em: <http://onlinelibrary.wiley.com/doi/10.1111/ gfs.1995.50.issue-3/issuetoc >. Acesso em: 5 jul. 2011. doi: 10.1111/j.1365-2494.1995.tb02314.x.

SANDERSON, M.A. et al. Growth and complexity of white clover stolons in response to biotic and abiotic stress. Crop Science, v.43, n.6, p.2197-2205, 2003. Disponível em: <https://www.crops.org/publications/cs/articles/43/6/2197>. Acesso em: 5 jul. 2011. doi: 10.2135/cropsci2003.2197.

SAS Institute. System for Information. Versão 8.2. Cary, 2001. 392p.

SINGH, D.K.; SALE, P.W.G. Defoliation frequency and the response by white clover to increasing phosphorus supply. I. Leaf dry matter yield and plant morphology responses.
Australian Journal of Agricultural Research, v.48, n.1, p.111-118, 1997a. Disponível em: <http://www.publish.csiro.au/ nid/40/paper/A96053.htm>. Acesso em: 5 jul. 2011. doi: 10.1071/A96053.

SINGH, D.K.; SALE, P.W.G. Phosphorus supply and the growth of frequently defoliated white clover (Trifolium repens L.) in dry soil. Plant and Soil, v.205, n.2, p.155-162, 1998. Disponível em: <http://www.springerlink.com/content/v27x085843320352/ fulltext.pdf $>$. Acesso em: 5 jul. 2011. doi: 10.1023/ A: 1004316726665 .

SINGH, D.K. et al. Phosphorus concentrations in the leaves of defoliated white clover affect abscisic acid formation and transpiration in drying soil. New Phytologist, v.146, n.2, p.249259, 2000a. Disponível em: <http://onlinelibrary.wiley.com/ doi/10.1046/j.1469-8137.2000.00644.x/pdf>. Acesso em: 5 jul. 2011. doi: 10.1046/j.1469-8137.2000.00644.x.

STRECK, E.V. et al. Solos do Rio Grande do Sul. Porto Alegre: EMATER/RS; UFRGS, 2002. 128p.

WILLIAMS, W.M. White clover taxonomy and biosestematics. In: BAKER, M.J.; WILLIAMS, W.M. (Ed). White clover. Wallingford: CAB International, 1987. p.323-342. 